\title{
Detection of HCV Core Protein in the Nuclei of Different Cell Types and in the Extracellular Matrix in Liver Biopsies from HCV-infected Patients
}

Viviana Falcón*, Nelson Acosta-Rivero*, Glay Chinea*, Jorge Gavilondo*, María-C de la Rosa*, Ivón Menéndez*, Santiago Dueñas-Carrera*, Ariel Viña*, Bienvenido Gra**, Miriam Noa***, Felix Alvarez*, Josefina Rodríguez*, Juan Morales*

*Biomedicine Division, Center for Genetic Engineering and Biotechnology., P.O. Box 6162, C.P. 10600, Havana. Email: viviana.falcon@cigb.edu.cu

**Institute of Gastroenterology, C.P. 10400, Havana; C.P. 10400, Havana;

***National Center for Scientific Research, P.O. Box 6990, Havana.

Understanding the mechanism of hepatitis $\mathrm{C}$ virus (HCV) pathogenesis is an important part of $\mathrm{HCV}$ research. Recent experimental evidence suggests that the $\mathrm{HCV}$ core protein ( $\mathrm{HCcAg}$ ) has numerous functional activities. These properties suggest that $\mathrm{HCcAg}$, in concert with cellular factors, may contribute to pathogenesis during persistent $\mathrm{HCV}$ infection [1]. On the other hand there is compelling evidence that $\mathrm{HCV}$ can infect immune cells, such as macrophages, B cells, and $\mathrm{T}$ cells [2]. Previously, we have detected HCcAg inside hepatocytes cells from patients with chronic HCV infection [3]; in this work the presence of HCcAg in other liver cell types (which are known to play a critical role in the response to liver injury leading to fibrosis) was examined. Liver needle biopsies from ten chronically HCV-infected patients were studied. As control the primary antibody was substituted by normal mouse serum. Samples of liver biopsies were fixed in $0.2 \%$ glutaraldehyde and $4 \%$ paraformaldehyde in $0.1 \%$ OsO4 in the same buffer, dehydrated in ethanol, embedded in lowicryl. Ultrathin sections were incubated with anti-HCcAg monoclonal antibodies (mAbs) recognizing the first 120 aa of $\mathrm{HCcAg}$. The ultrathin sections were examined with a JEOL JEM 2000EX transmission electron microscope. Immunostaining (arrows) with anti-HCcAg mAbs revealed the presence of this protein in various hepatic infiltrating or resident immune cells such as activated macrophages, Kupffer cells (Figure 1a), B lymphocytes (Figure 1b), antibody-producing plasmatic cells (Figure 1c), and mastocytes. Interestingly, HCcAg was immunolabeled (arrows) not only in the cytoplasm but also in the cell nucleus (N). In addition, HCcAg was shown to localise in Ito cells (IC) (Figure 1d) and fibroblasts (F) (Figure 1f) (Figure 2). Of note, specific immunolabeling (arrows) appeared widely distributed in the cytoplasm and also in the nuclei $(\mathrm{N})$. In the case of fibroblasts, $\mathrm{HCcAg}$ co-localized with collagen fibres (CF). Remarkably, HCcAg appeared to interact with large lipid droplets (LD) present in IC. Finally, the specific localization of HCcAg was also observed in the extracellular matrix (ECM) especially on the surface of CF (Figure 1g). It has been previously reported that $\mathrm{HCcAg}$ modulates the host immune responses [4]. We hypothesize from our data that it is possible that HCcAg may cause dysfunction of these $\mathrm{HCcAg-containing} \mathrm{immune} \mathrm{cells}$ and leads to immune function decline or in disorder, and this becomes more difficult for the host to clear intrahepatic HCV, so that the injure of hepatocytes persists. On the other hand, IC has a central role in the pathogenesis of chronic liver injury and ECM-digesting enzymes are primary mediators of this role. After activation, IC derives to fibroblast-like phenotype which is prominent in all fibrosis tissues and appears to be the source of most of the ECM elicited [5]. The presence of $\mathrm{HCcAg}$ in these cells specially localized in the nuclei may argue for a pathogenic role at this level. Besides, a role for HCcAg in leukocyte migration may also be mediated by direct or indirect binding to the collagen fibres present in ECM. Steatosis is a frequently occurring feature of HCV infection. The findings that $\mathrm{HCcAg}$ localized on the surface of large lipid droplets inside IC in HCV-infected 
patients support previous works which demonstrated that HCcAg contributes to steatosis [6]. Taken together, our present data suggest that the presence of $\mathrm{HCcAg}$ in the nuclei of various cells types which are known to play a critical role in the response to liver injury leading to fibrosis could be relevant for the pathogenesis of HCV. They also suggest the possibility of HCV replication in these cells as a mechanism of $\mathrm{HCV}$ persistence.

\section{References}

[1] K, Shimotohno. Seminars in Cancer Biol (2000) 10, 233.

[2] T, Laskus. Hepatology (1998) 28, 1398.

[3] V, Falcon, et al., Acta Microscopica (2001) Oct, 442.

[4] C. S, Hahn, et al., Virology (2000) 276, 126.

[5] G. W, McCaughan, et al., Immunological reviews (2000) 174, 172

[6] M, Okuda, et al., Gastroenterology (2002) 122, 366.

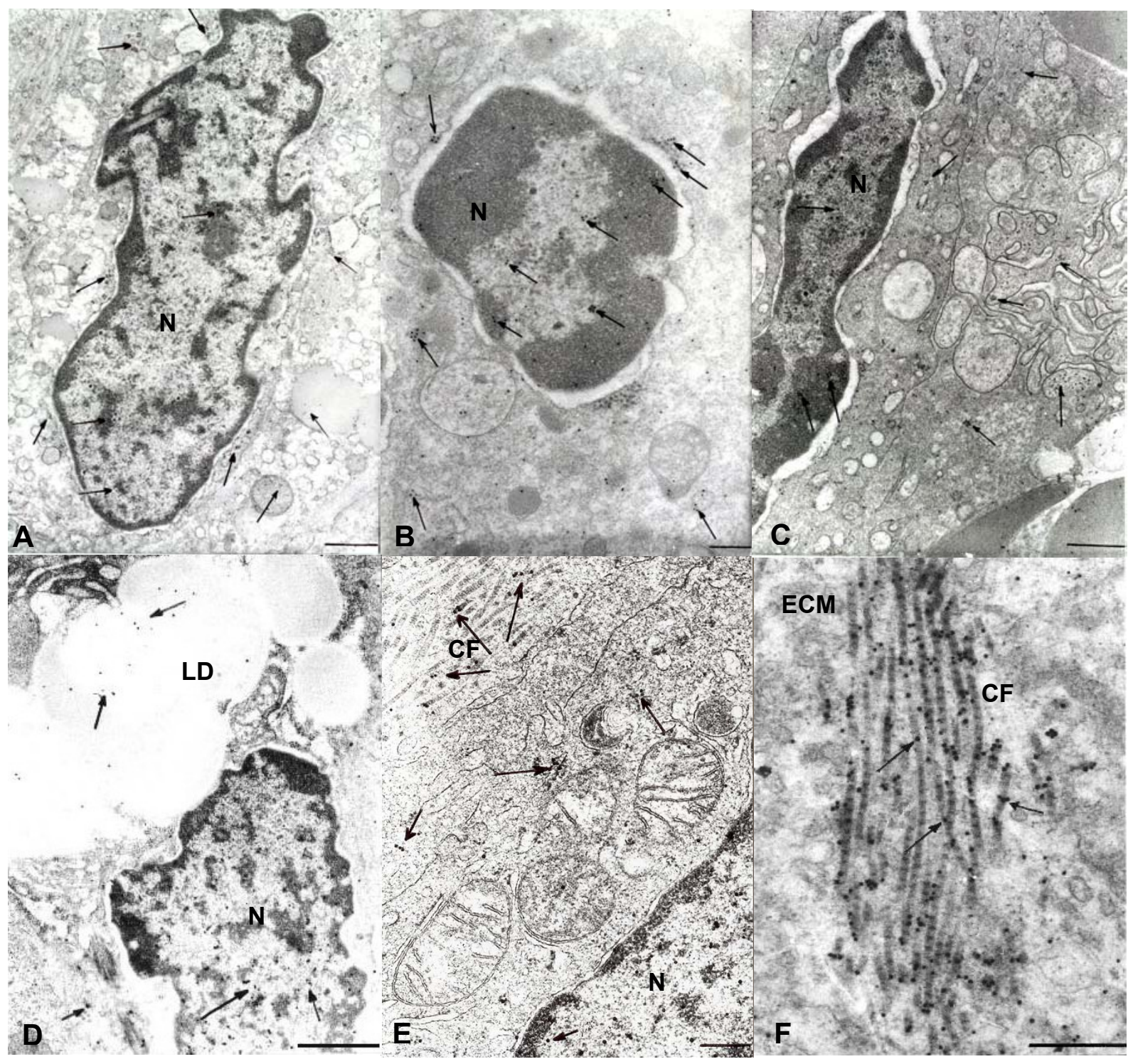

Figure 1. Bars $1 \mu \mathrm{m}$ in D; bar $=200 \mathrm{~nm}$ in $\mathrm{B}$; and bars $=500 \mathrm{~nm}$ in $\mathrm{A}, \mathrm{C}, \mathrm{E}, \mathrm{F}$. 